\title{
Ein Uterus gravidus aus der fünften Woche, der Lebenden entnommen.
}

\author{
Von \\ S. Gottschalk, \\ Berlin.
}

(Mit 8 Abbildungen anf Tafel IX.)

Wenn schon menschliche Eier aus der fünften Woche der Schwangerschaft immerhin zu den nicht alltäglichen Vorkommnissen zählen, so dürfte die Beobachtung eines solchen Eies in seiner natürlichen Lage in einem Uterus, der direct operativ der Lebenden entnommen wurde, zu den grossen Seltenheiten gehören, wenn nicht bis jetzt einzig dastehen.

Allgemein bekannt von solchen Präparaten ist der von Coste genau beschriebene Uterus gravidus von 40 Tagen, von dem v. Kölliker in seiner Entwickelungsgeschichte mehrere Abbildungen wiedergiebt. Allein so werthvoll dieses Präparat auch gewesen ist, so muss man doch einräumen, dass sich an ihm, da es in der Leiche gefunden wurde, bereits postmortale Veränderungen geltend gemacht hatten, welche unserem Präparate vollends abgehen, da ja hier das Organ mitten in seiner vollen Lebensfunction aus dem Körper entfernt wurde.

Ich habe Gelegenheit genommen, den betreffenden Uterus, dessen Beschreibung ich jetzt folgen lasse, in einer Sitzung der Berliner Gesellschaft für Geburtshülfe und Gynäkologie vom 14. Mai d. J., sowie auf der diesjährigen Naturforscherversammlung zu demonstriren.

Derselbe ist durch die wegen eines. Cancroid der Portio vaginalis ausgeführte Totalexstirpation von Herrn Dr. Landau, welchem ich für die Ueberlassung dieses seltenen Präparates, sowie für die Bereitwilligkeit, mit der er mir sein Privatlaboratorium zum Zwecke dieser Arbeit zur Verfügung stellte, meinen verbindlichsten Dank abstatte, gewonnen worden.

Der Vollständigkeit halber gebe ich die betreffende Krankenund Operationsgeschichte hier kurz wieder. 
Frau J., 32 Jahre alt, hat zwei Mal ausgetragene Kinder normal geboren und ein Mal vor sieben Monaten abortirt; sie ist stets regelmässig, wenn auch in letzterer Zeit etwas reichlich, menstruirt, zuletzt fünf Wochen vor der Operation. In den letzten Wochen hatte sich übelriechender, mehr oder weniger blutig gefärbter Ausfluss bei der Kranken eingestellt, ihre allgemeine Körperconstitution hatte sehr gelitten, sie war stark abgemagert und heruntergekommen.

Bei der Untersuchung liess sich feststellen, dass der Uterus etwa dem Beginne des zweiten Schwangerschaftsmonates entsprechend vergrössert war und jenes für die Schwangerschaft so charakteristische, weiche, teigige Gefühl darbot, dass die Wahrscheinlichkeitsdiagnose auf Schwangerschaft wohl gestellt werden konnte. Die Portio vaginalis war weich, aufgelockert und blutete bei der Einführung des Speculum stark; durch die leiseste Berührung mit dem Wattetupfer wurde die Blutung aufs äusserste verstärkt. Die Portio war ganz besetzt mit papillären, rosigen Wucherungen. Die mikroskopische Untersuchung ergab, dass es sich um ein Cancroid der Vaginalportio handle.

Von der supravaginalen Amputation, welche zweifellos zur gründlichen Entfernung der erkrankten Partien genügt haben würde, stand Herr $L$ a $n d a u$ zu. Gunsten der Totalexstirpation deshalb ab, weil nach der blossen Absetzung des Gebärmutterhalses die Narbe am schwangeren Uterus sicherlich weit eher zu einem Rückfalle neigt, und weil der so infolge des operativen Eingriffes wohl mit Sicherheit eingeleitete Abortus den normalen günstigen Heilungsverlauf gestört hätte.

Die am 6. Mai d. J. ausgeführte Operation verlief ganz glatt. Die heftige Blutung, welche auf die blosse Umschneidung des vorderen Scheidengewölbes erfolgte, die in ihrem Lumen sehr stark erweiterten Arteriae uterinae erhoben die Wahrscheinlichkeitsdiagnose auf Schwangerschaft während der Operation fast zur vollen Gewissheit. Das Organ wurde, wie wir das jetzt in der Regel zu thun pflegen, in situ herausgenommen und nicht vorher nach hinten umgestülpt.

Die Patientin machte eine ganz glatte Convalescenz durch und ist heute, wo etwa ein halbes Jahr nach der Operation verflossen ist, noch ohne jeden Rückfall und hat sich sichtlich erholt.

Das exstirpirte Organ wurde durch den Henle'schen Frontalschnitt von mir eröffnet, und nur dieser Form der Schnittführung 
ist es zu verdanken, dass das in der vorderen Wand eingebettete Ei - denn es war in der That ein schwangerer Uterus - beim Aufschneiden nicht verletzt wurde.

Leider hatte aber das Ei eine kleine Verletzung im Beginne der Operation dadurch davongetragen (vgl. Zeichnung 1), dass Herr Landau das von Brennecke angegebene Instrument zum Herunterziehen des Uterus angewandt hatte und der eine Haken desselben an einer Stelle in das Ei eingedrungen war, was, wie wir später sehen werden, den kleinen Embryo sogar seinen Kopf kosten sollte.

Ich möchte an dieser Stelle nicht verfehlen, eine Bemerkung über dieses von Brenuecke in das Instrumentarium der Totalexstirpation eingeführte Instrument einzuschalten. Eine Reihe von Fällen, in denen dasselbe zur Anwendung kam, hat mich fest überzeugt, dass dasselbe seinen Zweck vollkommen erfüllt; es gelingt leicht, mit demselben den Uterus gut herunterzuziehen, und es kann so vier Muzeux ersetzen. Allein es haftet ihm ein Nachtheil an, der ja leider sich gerade an unserem Präparate so geltend machte; derselbe äussert sich darin, dass die Innenfläche der Gebärmutterwand durch die beiden scharfen Haken, die zur Fixirung des Instrumentes in der Gebärmutter dienen, mehr oder weniger stark zerfleischt wird, so dass man sich nachher makroskopisch nicht leicht eine Vorstellung von dem ursprünglichen Aussehen der Gebärmutterschleimhaut machen kann. Wir sind daher in letzter Zeit wieder zu den Hakenzangen zurückgekommen.

Ich beginne mit der malkroskopischen Beschreibung unseres Präparates.

Die Figur 1 der beigegebenen Tafel zeigt uns den exstirpirten Uterus durch den Frontalschnitt nur theilweise eröffnet, der Schnitt auf der linken Längskante ist an ihm noch nicht durchgeführt; wir sehen das Ei dementsprechend etwas stärker gewölbt durch die eröffnete rechte Kante sich hervordrängen; dass dasselbe in der Natur mehr central in der Mitte der vorderen Wand, mit der grössten Wölbung nach der Mitte der hinteren Wand zu gerichtet gesessen hat, lehrt uns ein flüchtiger Blick auf Figur 2, welche uns den Uterus nach nachträglicher Eröffnung der linken Kante, von dieser aus gesehen, zeigt: wir sehen hier ganz deutlich, wie das Ovulum künstlich nach rechts hin gewaltsam verschoben ist. Soviel zur Erklärung, dass die Asymmetrie des Eies, wie sie nach den beiden Zeichnungen vorhanden zu sein scheint, 
eine künstliche, nachträglich durch die erwähnte zweizeitige Schnittführung entstandene, ist.

Das ganze Präparat ist so recht geeignet, uns eine genaue Vorstellung von den starken Veränderungen $\mathrm{zu}$ geben, welche der Uterus in den einzelnen Schichten seiner Wandung gerade in so früher Zeit der Schwangerschaft erfährt; wir können an ihm aber auch vortrefflich studiren, in welcher Weise das menschliche Ei sich in dem Uterus, seinem Bebrïtungsbette, einnistet, und welches die Beziehungen zwischen Ei und Gebärmutterwand, bezw. Gebärmutterschleimhaut sind. Der Bauchfellüberzug des Uterus ist allein unverändert geblieben, die Serosa überzieht als gleichmässig glänzender Ueberzug das schwangere Organ, dessen grösste Länge vom äusseren Muttermunde bis zur Mitte der oberen queren Kante, die Dicke der Wand mit eingerechnet, 8,9 cm, dessen grösste Breite in der Nähe des Fundus, gleichfalls die Dicke der Wandungen eingeschlossen, $6,6 \mathrm{~cm}$ beträgt.

Betrachten wir uns die Gebärmutterwand näher, so muss uns zunächst die Dickenzunahme der Muskulatur auffallen, die sich nicht nur am Fundus uteri, sondern auch an den unteren Partien des Organes, am Orificium internum und der Cervix, geltend macht. Ja, wenn auch am Fundus die Muskulatur 2,1 cm misst, so müssen wir doch zugestehen, dass die durch die Schwangerschaft bedingte Hyperplasie der Muscularis im Verhältniss am stärksten in der Höhe des unteren Eipoles, unmittelbar oberhalb des inneren Muttermundes, ausgesprochen ist; denn die Muskulatur ist hier nur um $0,4 \mathrm{~cm}$ weniger dick als am Gebärmuttergrunde. Es ist dies eine immerhin bemerkenswerthe Thatsache gegenüber dem Umstande, dass gerade hier unten das untere Uterinsegment sich ausbildet und also am hochschwangeren Organe die Wand hier gerade am dünnsten $z u$ sein pflegt.

Wenn wir aber genauer bestimmen sollen, wo denn die Muskulatur die grösste absolute Dicke erreicht hat, wo sie am dünnsten, so müssen wir die erstere Stelle etwas oberhalb der Mitte des Körpers suchen; denn hier hat diese Schicht schon die beträchtliche Dicke von $2,5 \mathrm{~cm}$ erreicht, die letztere aber auffallender Weise nach den Eileitermündungen verlegen; denn hier hat die Hyperplasie nicht gleichen Schritt gehalten, die Dicke der Muskulatur beträgt hier nur $1,2 \mathrm{~cm}$. Vielleicht hat diesielbe hier unter der ausserordentlich starken Wucherung der Schleimhaut leiden müssen, die umgekehrt gerade hier, also wohl auf Kosten der Muskulatur, ihre grösste Ausdehnung gewonnen hat: 
Nicht minder ist zu verkennen, dass auch die Mutterhals wand in ihrem muskulären Theile eine wenn auch verhältnissmässig geringere, aber desto gleichmässigere Hyperplasie erlitten hat. Ich konnte die Dicke dieser Schicht am inneren Muttermunde auf $1,2 \mathrm{~cm}$, am Orificium externum und besonders an der hinteren Lippe auf $\mathbf{1 , 5} \mathrm{cm}$ bestimmen. Vielleicht dürfte bei dem letzteren Verhalten die Neubildung an der Portio als ursächliches Moment in Betracht kommen; denn man müsste eigentlich erwarten, dass die Muskulatur am äusseren Muttermunde weniger dick oder mindestens nicht dicker sei, als am Orificium internum.

An Stelle der normalen Gebärmutterschleimhaut sehen wir das Innere der Höhle ausgekleidet mit der an einzelnen Stellen 0,4, an anderen $0,5 \mathrm{~cm}$ dicken Decidua vera, welche sich deutlich von ihrer Unterlage, dem basalen Theile der Gebärmutterschleimhaut, abhebt. Diese letztere Erscheinung ist besonders an der rechten Gebärmutterkante und weiter abwärts an dem freien, zackigen Rande der Decidua vera so stark ausgesprochen, dass hier ein deutlicher, schmaler Zwischenraum zwischen Vera und Gebärmutterwand sich ergiebt. Die deciduale Wucherung geht aber nicht auch auf den Gebärmutterhals über, sondern mit einem unregelmässig gezackten Rande endigt die Decidua vera frei gleich unterhalb des inneren Muttermundes, eine Erscheinung, auf die auch v. Kölliker aufmerksam macht, und die er als die charakteristische und gewöhnliche hinstellt.

Nun, die Mutterhalsschleimhaut an sich ist, wie gesagt, auch recht stiefmütterlich weggekommen, denn wir können nicht eine irgendwie bemerkbare Wucherung an ihr erkennen. Die Natur scheint, wie in der Regel, so auch hier, in erster Linie dem Zwecke und dem Bedürfnisse sich anzupassen und nur soweit Veränderungen an dem Organe zu erzeugen, als nach diesem Anpassungsprincipe unbedingt erforderlich sind. Welches ist denn der Zweck der Schleimhautwucherung? Die Natur will durch sie dem befruchteten Ei ein Bebrütungsbett schaffen, sie will gleichzeitig durch sie das befruchtete Ovulum im Gebärmutterkörper festhalten und so verhindern, dass dasselbe nach aussen treten und so verloren gehen kann. Wie weit muss aber die Schleimhautwucherung gehen, um diesen Zweck zu erfüllen? Offenbar genügt es, wenn diese Wucherung sich auf die Schleimhaut der eigentlichen Gebärmutterhöhle beschränkt; es ist für das so eingebettete Ei gleichgültig, ob nun die Schleimhaut des Mutterhalskanales in 
gleicher Weise Wucherung erfährt; die letztere ist nicht nothwendig; mit der vollendeten Wucherung der eigentlichen Gebärmutterschleimhaut ist die Bedürfnissfrage erledigt.

Ganz anders sieht es mit der Schleimhaut der linken Eileitermündung aus, hier setzt sich die Wucherung, soweit an dem Präparate noch vorhanden, auf die Eileiterschleimhaut fort, so dass die Wucherungszone der Schleimhaut hier die beträchtliche Breite von $1,9 \mathrm{~cm}$ gewonnen hat. Vielleicht dürfen wir in dem Umstande, dass die Mucosa der linken Tube sich an der Wucherung betheiligt hat, einen Wegweiser erblicken, der uns anzeigt, auf welche Weise denn das Ei in das Cavum uteri gelangt ist. Wahrscheinlich ist es durch diese linke Tube eingetreten und die Natur passte sich auch hier wiederum offenbar nur dem Zwecke und der Bedürfnissfrage an, wenn sie dem durch das Ostium uterinum tubae eintretenden Ovulum gleich hier bei seinem Eintritte ein Schleimhautbett geschaffen, das dasselbe festhalten und so ein Zusammentreffen von Ovulum und Sperma ermöglichen konnte. Denn wenn wir mit Wyder annehmen, dass die Befruchtung in der Regel im Uterus selbst stattfindet, so muss dieselbe in diesem Falle gleich in der Nähe der linken uterinen Eileitermündung erfolgt sein, denn hier ist die Fruchtkuchenstelle.

Von der dicken Decidua vera ist die dünne, nur $0,1 \mathrm{~cm}$ breite Decidua reflexa noch deutlich durch einen mit Schleim angefüllten Zwischenraum getrennt. Erst später legen sich diese beiden Häute unmittelbar an einander und verwachsen mit einander. Die Reflexa bildet die glatte Oberfläche des Eies, welches eine maximale Länge von $4 \mathrm{~cm}$ und eine maximale Breite von 2,4 cm hat, das aber die Gebärmutterhöhle noch nicht ganz ausfüllt, sondern mit seinem unteren Pole nicht ganz. $1 \mathrm{~cm}$ oberhalb des inneren Muttermundes endigt. Die Innenfläche der Decidua reflexa ist rauh, sie ist mit den feinen Zöttchen des Chorion ganz innige Verwachsungen eingegangen. Schön tritt besonders am oberen Eipole der Uebergang der Decidua vera in die Reflexa hervor; wir können hier feststellen, dass dieser Uebergang ein allmäliger ist.

Das Chorion laeve, welches, wie vorhin erwähnt, mit der unteren Fläche der Reflexa unzertrennbar durch seine $0,25 \mathrm{~cm}$ langen, noch ziemlich dicht bei einander sitzenden Zöttchen verbunden ist, ist nicht dicker als die Reflexa.

Die Zotten des Chorion frondosum sind bereits doppelt so. 
lang als die peripheren Zöttchen, sie messen bereits $0,5 \mathrm{~cm}$. Eine genauere Untersuchung der Fruchtkuchenstelle habe ich noch nicht vorgenommen, da mir daran gelegen war, das Präparat möglichst noch zu schonen.

Das Amnion ist durch den Bluterguss, welcher bei der erwähnten Verletzung des Eies in dessen Höhle statt hatte, zerstört worden und so für die makroskopische wie mikroskopische Untersuchung und Beschreibung leider verloren gegangen.

Nach der Eröffnung des Eies mittels eines Kreuzschnittes zeigt sich die ganze Höhle mit Blutgerinnseln ausgefüllt, zwischen denen der kleine, etwas platt gedrückte Embryo, freilich noch in situ, sich befindet, aber vollends decapitirt, welch letztes Unglück zweifelsohne beim Eindringen des Eingangs erwähnten scharfen Hakens geschehen sein musste. Der Embryo misst ohne Kopf $1,2 \mathrm{~cm}$; da der letztere einen senkrechten Durchmesser von 0,7 cm hat, so würde demnach die ganze Länge des Embryo 1,9 cm betragen. Der Rücken und das Schwanzende des Embryo sind stark gekrümmt, die oberen Extremitäten als 0,2, die unteren als 0,3 cm lange Stümmel angelegt. Der Kopf ist ebenfalls im geraden Durchmesser stark gedrückt worden, so dass das Gesicht ziemlich breit $(0,4 \mathrm{~cm})$ erscheint; die Mundspalte ist $0,25 \mathrm{~cm}$ breit, die zweite sehr schön ausgeprägte Kiemenspalte von der ersten 0,2 cm entfernt. Der Nabelstrang misst $\mathbf{1 , 3} \mathrm{cm}$.

Um die uns interessirenden wichtigsten Maasse zu vervollständigen, wäre hier die Länge des Mutterhalskanales noch nachzutragen, welche ich auf $3 \mathrm{~cm}$ bestimmen konnte.

Noch weit interessanter als das makroskopische Studium des Präparates dürfte die mikroskopische Betrachtung der Eihäute sein. Die Schnitte, welche im Folgenden dieser Betrachtung zu Grunde liegen, sind mit Pikrolithioncarmin gefärbt worden.

Ich beginne mit der mikroskopischen Beschreibung der Decidua vera und betrachte diese Haut zunächst bei schwacher Vergrösserung, welche uns das in der beigefügten Zeichnung wiedergegebene Bild liefert. Wir unterscheiden bei dieser Betrachtung eine äussere, der Gebärmutterhöhle zugewandte, Zellenschicht und eine innere Drüsenschicht.

Die Breite der Zellenschicht gestaltet sich verschieden, je nachdem die Drüsenschicht mehr oder weniger weit nach aussen sich erstreckt. Soviel aber lässt sich von der Zellenschicht schon. bei dieser schwachen Vergrösserung mit Gewissheit feststellen, 
dass die einzelnen Elemente dieser Schicht ziemlich dicht und innig an einander liegen müssen, denn das Auge kann keine Lücken hier entdecken.

Ganz anders die Drüsenschicht! Sie ist der herrschende Theil, sie ist es, welche dem ganzen Schnitte sein so charakteristisches Gepräge, der ganzen Membran ihren richtigen Namen verleiht: Denn eine siebförmige Membran mit verschieden grossen Löchern ist es, als welche wir diesen inneren und grösseren Theil der Decidua vera ansprechen müssen. Die in ihrem Lumen stark erweiterten Drüsen sind durch Zwischenbalken von einander getrennt, die nach innen zu sich stark verjüngen, um zuletzt ganz zu schwinden, so dass hier Drüse an Drüse grenzt und sich am innersten Theile der Drüsenschicht erst recht geltend macht, wie sehr gerade diese Haut den Namen "Siebhant" verdient.

Betrachten wir jetzt die Zellenschicht bei starker Vergrösserung, so fällt uns auf, dass hier schöne, grosse, ovale und runde Zellen mit Kernen und Kernkörperchen so dicht an einander gelagert sind, dass für ein bindegewebiges Zwischengewebe nur sehr spärlicher Raum übrig geblieben ist. Diese Zellen sind scharf umgrenzt, theilweise gekörnt und schwanken in ihrer Grösse zwischen der weisser Blutkörperchen und der von Riesenzellen; wie die Zellen selbst, so fallen auch deren Kerne durch ihre Grösse auf und durch die Intensität, mit der sie den Farbstoff in sich aufgenommen haben. Als charakteristisch für diese Schicht möchte ich die Ineinanderschichtung der einzelnen Zellen besonders am äusseren Rande hinstellen; die Regelmässigkeit der Anordnung dieser Zellen verfehlt nicht, dem Gesammtbilde etwas für das Auge sehr Angenehmes zu verleihen.

Die ganze Zellenschicht dürfen wir, da sie die Drüsenschicht nach aussen überzieht, als eine Deckschicht ansehen und vielleicht am ehesten dem geschichteten Pflasterepithel an die Seite stellen, ohne aber in den Fehler zu verfallen, zu glauben, dass nun ein wirkliches Pflasterepithel hier vorliegt. Dazu ist die Schicht zu breit, es fehlt ihr vor allen Dingen aber die Abplattung der obersten Zellenlagen, die ja für das Pflasterepithel so charakteristisch ist; ja im Gegentheil, wir werden hier sogar die Zellen der äussersten Lagen als die schönsten anerkennen müssen, die hinsichtlich der Grösse und der scharfen Umgrenzung vor den unter ihnen liegenden nicht zurückstehen. Mehr nach der Drüsenschicht zu geht das innige Ineinandergreifen und die dichte Lagerung der schönen 
Deciduazellen allmälig verloren, so dass hier ein Zwischengewebe bindegewebiger Natur als die bindende Kittsubstanz sich deutlich geltend macht.

Den gleichen Bau zeigen auch die Zwischenbalken, welche weiter nach innen die Grenzscheiden der Drüsen bilden; auch sie bestehen aus kernhaltigen Zellen von verschiedener, aber vorwiegend rundlicher Gestalt von der Grösse weisser Blutkörperchen bis zu der von wirklichen Riesenzellen, zwischen denen eine bindegewebige Grundsubstanz zu erkennen ist.

Die Capillaren der Drüsenschicht sind strotzend mit Blut gefüllt und durchweg stark erweitert. Ungehener blutreich muss diese Membran sein; denn zahlreiche Blutaustritte, welche theils in die Nachbarschaft der Capillaren, theils in die erweiterten Drüsenöffnungen hinein erfolgt sind, geben hiervon das beredteste Zeugniss ab. Vielleicht sind diese Extravasate erst während der Operation entstanden; wie dem auch sei, die Folgen eines starken Blutaustrittes sehen wir zweifelsohne in dem blutigen Inhalte der Drüsen.

Wenn wir bei schwacher Vergrösserung von dem mittleren Theile der Pars spongiosa sagten, dass die Zwischenbalken hier geschwunden seien und Drüse an Drüse grenze, so nöthigt uns die starke Vergrösserung, unsere Aussage dahin zu mildern, dass diese Drüsenöffnungen doch nicht so unmittelbar an einander grenzen; allerdings ist der Zwischenraum zwischen ihnen ein sehr kleiner, allein er ist doch immerhin da und wird von jenen schönen Deciduazellen, die durch spärliches Bindegewebe zusammengehalten werden, ausgefüllt. Musste die Regelmässigkeit in der Anordnung der einzelnen Elemente bei der Betrachtung der äusseren Zellenschicht unser Wohlgefallen erwecken, so vermisst unser kritisches Auge auch hier keineswegs die in der Natur sich ja bis in das Kleinste offenbarende Ordnung. Denn hier haben die schönen kernhaltigen Zellen das Bestreben, sich um fünf bis neun zusammenliegende Genossinnen als ihren Mittelpunkt concentrisch herumzulagern, so dass daraus Kreise von Deciduazellon entstehen, die, wenn auch nicht ganz regelmässig, so doch im Ganzen and Grossen nicht zu verkennen sind. Bindegewebsfasern bilden den Kitt, welcher diese einzelnen Kreise der Zwischensubstanz zusammenhält; dieselben sind freilich im Centrum dieser Kreise nur spärlich, mehr nach der Peripherie zu jedoch stärker vertreten. Gehen wir aber tiefer in die spongiöse Schicht hinein, so wird 
auch diese Zwischensubstanz auf ein Kleinstes beschränkt, ja, es macht hier fast den Eindruck, als ob ursprünglich Drüse an Drüse angrenzt und nur eine Art entzündlicher Infiltration zwischen die einzelnen Drüsenlumina erfolgt sei. Denn nicht jene schönen Deciduazellen erblicken wir hier zwischen den einzelnen Drüsen: Rundzellen, jedoch mit Kernen und theilweise wenigstens von gekörntem Aussehen, bedeutend kleiner als die Deciduazellen, bilden eine nur dünne Zwischenlage, der eine bindegewebige Grundsubstanz völlig abgeht. Es ist das ein wesentlicher Unterschied gegenüber dem Bau der Zwischenbalken des äusseren Theiles der spongiösen Schicht, den ich nochmals in dem Hinweise auf den Grössenunterschied der Zellen und den Mangel an bindegewebiger Grundsubstanz in der zuletzt beschriebenen innersten Lage der spongiösen Schicht hervorheben möchte.

Es hat fast den Anschein, als ob in der spongiösen Schicht das Hauptbestreben des Wachsthumes darauf hinausginge, die Räume der präexistirenden Drüsen auf das äusserste zu erweitern und auszubuchten. Es kann uns daher nicht wundern, wenn dort, wo dieses Bestreben am deutlichsten zu Tage tritt, in dem innersten Theile der spongiösen Schicht, gleichsam kein Raum für ein interglanduläres Zwischengewebe, besonders für grosse Deciduazellen, geblieben ist, wenn ferner auch das innere Auskleidungsepithel der Drüsen, sich beugend dem von innen auf es einwirkenden Drucke, an Höhe eingebüsst hat. Die schönen hohen Cylinderzellen, welche wir an der normalen Gebärmutterschleimhaut bewundern und die an der äusseren Grenze der spongiösen Schicht grösstentheils noch erhalten sind, haben sich abgeplattet und einen mehr cubischen Charakter angenommen. Nur die Zellkerne allein scheinen unter der Ausdehnungskraft der Drüsen nicht gelitten zu haben, denn überall, an allen Theilen der Drïsenschicht fallen sie uns durch ihre Schönheit auf. Den Verlust der Flimmerhaare aber müssen auch die Drüsenepithelien des äusseren Theiles der spongiösen Schicht mit denen des inneren theilen; nirgends mehr können wir Flimmerhaare wahrnehmen. Einen Ersatz für diesen Verlust scheint den Drüsenepithelien der inneren Spongiosa die Natur haben geben zu wollen in einer einschichtigen basalen Unterlage von runden und auch ovalen kernhaltigen Zellen, denen das eigentliche cubische, früher cylindrische Auskleidungsepithel aufsitzt. Wir haben so hier ein ähnliches Bild wie beim Epithel des Froschdarmes, und wenn wir dort, wo den grossen Cylinderzellen an ihrer Basis die kleinen Ersatzzellen anliegen, von einem geschichteten Cylinderepithel 
sprechen, so dürfen wir mit demselben Rechte das Auskleidungsepithel der Drüsen hier als geschichtetes cubisches Epithel bezeichnen.

Dass aber die beschriebene Abplattung des Epithels die Folge eines Druckschwundes ist, bei welchem der von zwei benachbarten Drüsenräumen auf einander ausgeübte Druck das wirksamste Motiv ist, lehrt ein Blick auf die beigegebene Zeichnung, aus der hervorgeht, dass an einzelnen Stellen nicht nur. eine Verdünnung der Scheidewand eingetreten, sondern gar mehrere benachbarte Drüsen mit einander verschmolzen sind zu einem unregelmässig begrenzten Raume, der als Ueberrest der untergegangenen Finrichtung nur einzelne, in das Lumen vorspringende Leisten von Drüsenepithel erkennen lässt.

$\mathrm{Ob}$ in dem Drüsenraume ein Inhalt vorhanden ist, der sich in der ersten Zeit der Schwangerschaft so schnell vermehrt, dass dadurch die Ektasie der Drüsen, ihr Bestreben, sich aufs äusserste zu erweitern und zu schlängeln, bedingt wird, wäre wohl möglich, lässt sich aber nicht mit Bestimmtheit sagen. Bisher sind diese Drüsen stets leer gefunden worden, und v. Kölliker spricht in seiner Entwickelungsgeschichte die Vermuthung aus, dass diese Drüsen im Leben wohl Flüssigkeit führten, über deren Natur keine Thatsache Aufschluss geben könnte. Von vornherein sollte man erwarten, dass gerade unser Präparat, welches doch möglichst lebende Verhältnisse wiedergiebt, uns hätte darüber aufklären können.

Ich habe bereits erwähnt, dass eine Anzahl der Drüsenräume aufs höchste mit Blut ausgefüllt sind und habe dies als die Folge eines Blutergusses, der möglicherweise erst während der Operation eingetreten sei, angesehen.

Es hat für mich etwas sehr Gezwungenes, etwa anzunehmen, dass diese Drüsen nun während des Lebens zu dieser frühen Zeit der Schwangerschaft Blut führten und so gleichsam Bluthöhlen darstellten.

Wenn v. Kölliker weiter gefunden hat, dass die Vergrösserung der Drüsen sich mehr auf die mittleren und oberen Partien beschränkt, während die blinden Enden nur wenig zunehmen, so kann ich dem im Ganzen und Grossen zustimmen, indem ich mich so ausdrücke, dass die Vergrösserung der Drüsen sich vorzugsweise a uf die mittleren und oberen Theile erstreckt, dass aber auch eine deutliche Zunahme sowohl in die Breite als in die Tiefe an den blinden Enden nicht zu verkennen ist: der Drüsengrund ist bedeutend weiter 
und ragt tiefer in die hyperplastische Muskulatur hinein, als beim nicht schwangeren Uterus. Auch gewinnt es den Anschein, als ob an der Basis vom Innern der Drüse aus ein entzündlicher Reiz auf die Umgebung ausgeübt worden sei, als dessen Antwort wir die runden Granulationszellen, welche den Drüsengrund dicht umlagern, anzusehen hätten.

Wenn man allgemein annimmt, dass die Decidua vera nichts anderes sei, als die hypertrophische Gebärmutterschleimhaut, so glaube ich, dass diese Anschauung einer Einschränkung bedarf. Es ist zweifellos, dass die Vera aus der Gebärmutterschleimhaut durch Wucherung der letzteren hervorgeht. Stellte sie aber schliesslich nichts anderes dar als eine hypertrophische Gebärmutterschleimhaut, so müsste sich diese Hypertrophie an den einzelnen Elementen der Decidua vera auch kundgeben; wir müssten dieselben Gewebe, wie bei der Gebärmutterschleimhaut, auch hier an der Vera in vermehrter, d. h. hypertrophischer Weise nachweisen können. Mit anderen Worten, diese Hypertrophie der Gebärmutterschleimhaut müsste sich entweder als eine interstitielle, das Bindegewebe allein betreffende, oder als eine glanduläre, oder aber als eine gleichzeitig interstitielle und glanduläre ausweisen. Im ersteren Falle müsste die bindegewebige Zwischensubstanz an der Decidua vera verbreitert und vermehrt sein; wir haben aber gesehen, dass gerade im Gegentheil, wenn die Zwischensubstanz an einzelnen Stellen gar auf ein geringstes Maass beschränkt war, diese nicht einmal überhaupt Bindegewebe führte, sondern nur aus zelligen Elementen gebildet wurde. Also als die durch interstitielle Wucherung hypertrophische Gebärmutterschleimhaut ist die Vera nicht anzusehen.

Damit ist aber auch schon die dritte Möglichkeit, dass die Vera eine gleichzeitige Vermehrung des glandulären und interstitiellen Gewebes der Gebärmutterschleimhaut in sich darstelle, als nichtig erklärt; denn wenn wir nachgewiesen haben, dass die Vera überhaupt weniger Bindegewebe führt, als die normale Gebärmutterschleimhaut, wir also einen Bindegewebsschw und bei ihr finden gegenüber ihrer Mutterschleimhaut, so kann nur noch ein vermehrtes Drüsenwachsthum, falls es an der Decidua nachweisbar ist, den Namen der Hypertrophie, und zwar besonders der partiellen Hypertrophie rechtfertigen. Aber auch dem ist nicht so; die Zahl der Drüsen ist keineswegs vermehrt, es ist keine Drüsenneubildung hier eingetreten; die einzelne Drüse hat eine grössere Weite angenommen, der Drüsenraum ist erweitert, und wenn wir dann 
noch gesehen haben, dass dies hier und da sogar auf Kosten der Selbstständigkeit geschieht, indem mehrere Drüsen in eine mit einander verschmelzen, so erwächst daraus von vornherein eine numerische Abnahme der Drüsen. Aber auch die starke Ektasie der Drüsen ist keineswegs das Ergebniss einer erhöhten Wachsthumskraft, wie wir es bei der glandulären Entzündung der Gebärmutterschleimhaut sehen; dem steht die Abplattung des Auskleidungsepithels entgegen, das doch offenbar unter einem Drucke, der von innen heraus auf dasselbe wirkte, gelitten hat; die Umwandlung des schönen Cylinderepithels in ein geschichtetes cubisches müssen wir als die Folge eines Druckschw undes ansehen. Warum sollte denn, wenn hier nur ein reines Mehr als Folge einer Nutritia nimia vorläge, gerade das Drüsenepithel an seiner Schönheit und Höhe einbüssen, also eine Atrophie erleiden?

Dazu kommt dann noch ein neuer Gesichtspunkt in den für die Decidua vera specifischen Deciduazellen, die wir vergeblich in der Gebärmutterschleimhaut suchen. Ich lasse dabei dahingestellt, ob diese schönen Zellen aus Bindegewebszellen oder aus dem Epithel der Gebärmutterschleimhaut genetisch hervorgegangen sind; für die erstere Anschauung spräche wohl der Umstand, dass das interglanduläre Gewebe grösstentheils auch aus diesen Zellen gebildet wird; jedoch ist nicht ausgeschlossen, dass beide Möglichkeiten gleichzeitig zu Recht bestehen.

Auf Grund der angeführten Thatsachen glaube ich berechtigt zu sein, vor der gebräuchlichen Erklärung der Decidua vera, ,dass sie nichts anderes darstelle, als die hypertrophische Gebärmutterschleimhaut", warnen zu müssen, soweit sich diese Bestimmung auf die histolo gis che Beschaffenheit der Decidua vera erstreckt. Ich gebe zu, dass man makroskopisch die Vera, als durch Hyperplasie aus der Gebärmutterschleimhaut hervorg egangen bestimmen kann, indem man dabei im Auge hat, dass die Vera durch Wucherung der Gebärmutterschleimhaut gebildet wird und auch histologisch alle Elemente der Vera aus präexistirenden Elementen der Schleimhaut entstanden sind. Aber deshalb, weil sie aus diesen hervorgegangen sind, brauchen sie ihnen nicht gleich zu sein, und dass in der That die Decidua einen anderen Bau zeigt, als die normale Gebärmutterschleimhaut, glaube ich im Vorhergehenden hinlänglich nachgewiesen $\mathrm{zu}$ haben. Und diese letztere Thatsache ist es, welche verbietet, die Decidua vera als hypertrophische Gebärmutterschleimhaut histologisch zu bestimmen. 
Ich betrachte jetzt bei starker Vergrösserung einen Schnitt, welcher der Uebergangsstelle der Decidua vera in die Reflexa entnommen ist. Die schönen Deciduazellen werden hier länglich, sie strecken sich, mit ihnen nimmt der frühere runde Kern auch eine ovale und schliesslich längliche Gestalt an. Diese Veränderung in der Structur der Zellen ist besonders in der äussersten Zone ausgesprochen; an der Grenze gegen die Drüsenschicht hin sind die Zellen noch mehr oder weniger unverändert geblieben. Sie stechen so stark gegen die Zellen der äusseren Zone ab, in deren Umwandlung ich etwas für diese Uebergangsstelle Charakteristisches erblicken möchte: Die erwähnten länglichen Zellen, die auch in der Breite nochetwas zugenommen haben und den Charakter grosser Spindelzellen mit spitzen Ausläufern tragen, sind sonst nirgends an der Vera vorhanden.

Die Drüsenschicht ist hier bereits stark vermindert, sie ist bedeutend schmäler geworden; nur noch wenige vereinzelte Drüsen sind an dem Schnitte wahrzunehmen, die jedoch hinsichtlich ihres Baues unverändert geblieben sind.

Gehen wir nun zu dem histologischen Studium der Decidua reflexa über und legen wir unserer Betrachtung einen senkrechten Schnitt zu Grunde, der durch alle Schichten der Reflexa und des mit ihr verwachsenen Chorion geht.

v. Kölliker sagt in seinem Lehrbuche der Entwickelungsgeschichte von der Reflexa, dass deren Gewebe so ziemlich mit dem der Vera.übereinstimmt. Ich bedaure, mich dieser Ansicht für diese frühe Zeit der Schwangerschaft nicht anschliessen zu können; ein Blick auf die beigegebene Zeichnung dürfte mit Leichtigkeit dies hinlänglich begründen. Vor allen Dingen ist es der Mangel an jeglichem Drüsengewebe, welcher uns in dieser Anschauung nur bestärken konnte; es fehlt überhaupt aber zu dieser Zeit nicht weniger als Alles, um einer Aehnlichkeit zwischen der Structur der Vera und Reflexa Platz geben zu können.

Ich unterscheide an der Reflexa von aussen nach innen, dem Chorion zu gehend, folgende Schichten:

1) Die,Epithelschicht der Reflexa.

Dieselbe besteht aus einer einfachen Lage cubischen Epithels auf einer bindegewebigen Grundlage, in der man Bindegewebszellen eingelagert findet (vgl. Fig. $5 a$ ).

2) Eine subepitheliale Bindegewebsschicht. 
Während die bindegewebige Grundlage des cubischen Epithels aus parallel verlaufenden Fasern besteht, können wir hier eine ausgesprochene Kreuzung der Fibrillen feststellen, so dass ein Netzwerk daraus sich ergiebt, in dessen Maschen grosse Zellen mit einem oder zwei schönen Kernen liegen. Diese Zellen sind bald mehr rund, bald mehr länglich und haben dieselbe Grösse wie die Deciduazellen der Vera; ich möchte sie deshalb auch als solche ansprechen (Fig. $5 b$ ).

3) Die von der vorigen Schicht durch eine Membrana limitans (elastica?) getrennte Substantia propria Reflexae (Fig. 4c).

Ganz aus embryonalem Schleimgewebe zusammengesetzt, weist diese ganze Schicht keine Zellen auf und entbehrt jeglicher geformter Elemente. Nur in unmittelbarer Nähe der Zotten, welche in diese Schicht hineingewachsen sind, gewinnt es den Anschein, als ob hier feinste, bindegewebige Fibrillen vorhanden wären (Fig. 5c). Die Richtung, in welcher diese fibrillären Elemente verlaufen, scheint gewissermaassen bestimmt zu werden durch die Wachsthumsrichtung der Chorionzotten, indem diese Bindegewebsfasern das Bestreben haben, die convexen Endigungen der Zotten dicht und möglichst concentrisch zu umlagern.

Die Breite der einzelnen Schichten der Reflexa nimmt stetig von aussen nach innen in der Weise zu, dass die epitheliale Schicht am schmälsten, die Substantia propria am breitesten ist.

Mit dieser kurzen Beschreibung dürfte der ganze histologische Bau der Reflexa schon wiedergegeben sein und aus ihr hinlänglich hervorgehen, warum ich oben meine Anschauung über diese Membran dahin ausdrückte, dass die Reflexa zu dieser frühen Zeit der Schwangerschaft einen von der Veradurchaus verschiedenen Bau zeige.

Wenn E. H. Weber und Sharpey in der Reflexa dieselben Drüsenmündungen gefunden haben, welche auch die Vera besitzt, und deshalb die Reflexa der Gebärmutterschleimhaut beizählen wollen, wenn auch Coste diese Ansicht durch seine umfassenden Untersuchungen zu stützen scheint, so kann ich hier nur wiederholen, dass es mir nicht möglich war, an der Reflexa unseres Präparates auch nur eine einzige Drüse nachzuweisen. Es bleibt danach nur die Möglichkeit bestehen, dass die Gebärmutterdrüsen sich überhaupt nicht an der Bildung der Reflexa betheiligen oder aber bereits in der fünften Schwangerschaftswoche vollends verschwunden sind; 
das letztere scheint mir die grössere Wahrscheinlichkeit für sich zu haben.

Wenn man vielfach annimmt, dass die Verbindung zwischen Decidua reflexa und dem Chorion laeve eine lockere sei, so kann ich dem gegenüber nur wiederholt erwähnen, dass diese beiden Häute hier so innig verwachsen sind, dass es auf dem mikroskopischen Schnitt den Anschein gewinnt, als ob beide Membranen eins wären.

Die Beschreibung des Chorion laeve müssen wir mit dem Theile derselben beginnen, welcher am weitesten nach aussen in die Substantia propria deciduae reflexae vordringt, mit den $\mathrm{Z}_{0}$ t te $\mathrm{n}$.

Die Betrachtung der beigegebenen Zeichnung lehrt, dass diese Zotten stets zu zweien angeordnet, also paarweise mitten in die Schleimschicht der Reflexa hineingewachsen sind, und zwar so, dass die Fasern der einen Zotte in die der zugehörigen zweiten übergehen und umgekehrt; man könnte also auch hier von einer Zotte mit zwei Endausbuchtungen sprechen.

1) Das Chorion laeve zeigt als oberste Lage eine $\mathrm{Z}$ elle $\mathbf{n}$ schicht (Fig. 6d). Ziemlich grosse, schön aufgebaute Zellen mit hübschem Kerne finden sich in zwei Lagen angeordnet, die bald mehr kreisrunde, bald mehr ovale, bald auch spindeiförmige Gebilde in feinfaseriger Grundsubstanz darstellen. Wir werden nicht fehl greifen, wenn wir auch diesen den Charakter der Deciduazellen zusprechen. An der äusseren Basis der Zotten, wo diese Zellenlage anfängt, ein Epithel für die Zotten abzugeben, können wir drei bis vier Lagen solcher Zellen entdecken, von denen eine jede in einem vorgebildeten bindegewebigen Maschenraume gelegen ist. Wenn Friedländer, Kundrat und Engelmann diese Zellenschicht als der Decidua reflexa angehörig beschreiben, so kann ich dies nach dem Gesagten mit v. Kölliker nur bestreiten und mit dem letzteren Autor die Zellenschicht als das Epithel des Chorion laeve ansehen. Betrachten wir das Epithel der Zotten bei starker Vergrösserung, so können wir feststellen, dass die eigentliche Zotte von einem feinfaserigen, dichten Gewebe gedeckt wird, an dessen Oberfläche eine ein- bis zweifache Lage einkerniger Zellen hinzieht. Es ist an einzelnen Stellen schwierig, nachzuweisen, dass diese schönen Zellen alle untereinander zusammenhängen; es wird das Auge an einzelnen Stellen sich vergeblich bemühen, diesen Zusammenhang zu erkennen. Zum Begriffe einer Epitheldecke ist aber eine solche Verknüpfung wohl ein 
unumgängliches Erforderniss. Ich erkenne die beschriebene Deckschicht an den Zotten an. Auf Grund der vorhin erwähnten Thatsache, dass die Epithelzellen über den Zotten nicht überall zusammenhängen, kann ich jedoch nicht v. Kölliker beipflichten, wenn er annimmt, dass die Zotten aus Zellen gebildet seien und nichts als hohle Auswüchse derselben darstellten, in welche der bindegewebige Theil erst nachträglich hineinwachse. Mir scheint der Vorgang der Zottenbildung ein anderer zu sein; ehe ich jedoch denselben schildern kann, ist es nothwendig, dass ich vorher die auf die Zellenschicht folgende Gewebslage beschreibe.

2) Eine aus feinsten, hellen, das Licht stark reflectirenden Fasern bestehende Schicht ist es, welche, unter der Zellenschicht gelegen, die zweite Lage des Chorion laeve bildet. Diese Fasern sind für den Farbstoff nicht sehr zugänglich, ja sie färben sich so schwer, dass an dem sonst gut gefärbten Präparat diese feinfaserige Zone durch ihren hellen Glanz hervortritt. Die einzelnen Fasern laufen parallel und in horizontaler Richtung, bis sie an einer Zotte angelangt sind; hier bilden sie dann eine nach oben stark convexe Halbwelle mit steil verlaufendem Wellenbogen; jedoch ist das Wellenthal nicht so tief, dass die Fasern dasselbe Niveau wieder erreichten, welches sie vordem hatten; bei starker Vergrösserung differirt die Höhe wohl um ca. $1 \mathrm{~cm}$ : Der aufsteigende Bogen der Welle ist länger als der absteigende. Dies ist ja auch sehr erklärlich; die Fasern sollen noch eine neue zweite Welle beschreiben, bevor sie auf ihre ursprüngliche Tiefe wieder hinabsteigen können. Zu diesem Zwecke nehmen sie, sobald sie das Wellenthal erreicht haben, auf eine kurze Strecke eine horizontale Richtung ein und steigen dann zum zweiten Wellenberge an, um dann schliesslich auf der anderen Seite zur' ursprünglichen Ausgangshöhe hinabzusteigen. Demzufolge muss also hier gerade umgekehrt der aufsteigende Bogen um dieselbe Differenz kürzer sein als vorhin der absteigende. Während dieses ganzen Verlaufes ist die Richtung der einzelnen feinen Fasern zu einander stets eine parallele zu nennen.

Die Breite der Wellën ist eine nur geringe, aber verschieden in der Weise, dass sie bei der innersten Faser, i. e. am tiefsten gelegenen, am kürzesten, bei der äussersten, i. e. am höchsten gelegenen, am grössten ist. Daraus entsteht dann eine nur schmale Lücke zwischen dem aufsteigenden und absteigenden Wellenbogen, welche nach abwärts allmälig breiter wird.

Dadurch, dass in der vorhin beschriebenen Weise alle Fasern 
verlaufen, haben wir schliesslich jenes Gebilde vor uns, welches wir Zotte oder richtiger ein Zottenpaar nennen. Die mikroskopische Betrachtung zeigt denn auch, dass das eigentliche Grundgewebe einer Zotte aus jenen feinen Fasern der vorhin erwähnten Schicht besteht, und ich trage deshalb kein Bedenken, diese Schicht ihres morphologischen und physiologischen Charakters wegen als "feinfaserige Zottenschicht" zu bezeichnen (Fig. 6e).

Der Vorgang der Zottenbildung gestaltet sich demnach nach unserer Ansicht so, dass diese feinfaserige Zottenschicht in der beschriebenen Weise je zwei Zotten bildet und während dieses charakteristischen Wachsthums die ihr aufliegende Zellenschicht einfach mechanisch zwingt, ihrer Wachsthumsrichtung zu folgen; das Epithel der Zotte, welches aus diesem Vorgange hervorgeht, ist eben nichts anderes als die erwähnte Zellens chicht des Chorion. Und da in diesem die beschriebenen grossen kernhaltigen Zellen nicht unmittelbar aneinanderliegen, so kann es auch nicht Wunder nehmen, wenn wir auf der Oberfiäche einer solchen Zotte auch nicht eine aus zusammenhängenden Zellen gebildete Epitheldecke erblicken, sondern hier und da blos die bindegewebige Grundsubstanz der Zellensehicht die Zotte iiberzieht. Später mag vielleicht durch Theilung der Zellen eine zusammenhängende Zellenschicht entstehen, vorläufig ist sie noch nicht da. Aus dem Gesagten wird sich wohl zur Genüge erklären, warum ich mich der Ansicht, dass die Zotten primär hohle Zellenauswüchse darstellen, nicht anschliessen kann.

3) Nach abwärts von der feinfaserigen Zottenschicht kommen wir auf ein dichtes, längsgestreiftes Gewebe, das den Charakter von elastischem Gewebe trägt, und da die einzelnen Fasern meist einander in der Längsrichtung parallel verlaufen, so kann diese Schicht wohl elastische Längsfaserschicht genannt werden (Fig. 6f). Die Sohicht hat ungefähr dieselbe Breite wie die vo rige. Dadurch, dass die Fasern dieser Schicht stets in derselben Ebene verlaufen, verbleibt zwischen je zwei Zotten ein Raum von ungefähr folgender Gestalt:

(vgl. Fig. 6k).

Die oberen kleinen spitzwinkeligen Dreiecke an dieser Figur stellen den freien Raum im Innern einer Zotte dar, die beiden seitlichen Winkel werden von der feinfaserigen Zottenschicht und 
der elastischen Längsfaserschicht gebildet. Die obere horizontale Grenze ist die Umschlagsstelle einer Zotte in die zugehörige zweite; die Basis der Figur bildet die elastische Längsfaserschicht.

Dieser Raum, der keine eigene Wandung aufzuweisen hat, ist ganz angefüllt mit Blut und stellt somit einen wandungslosen Blutraum dar, so dass also die Zotten in ihrem Innern von. freiem Blute umspült werden. Nur vereinzelte Fasern der elastischen Schicht ziehen quer durch den unteren Theil dieses Blutraumes und bilden das einzige heterogene Element in diesem Blutraume. Die letzterwähnte elastische Schicht zeigt hier und da spärliche Bindegewebszellen in ihrem Innern, so dass sie eine auffallende Aehnlichkeit mit der bindegewebigen Grundsubstanz des Epithels der Reflexa gewinnt.

4) Durch eine Membrana limitans von der vorigen Schicht getrennt, folgt dann die gefässhaltige Bindegewebsschicht des Chorion, die man, in Anbetraoht des Umstandes', dass die Gefässe den Haupttheil dieses Gewebes bilden, wohl noch besser als bindegewebige Gefässschicht (Fig. $7 h$ ) bezeichnen dürfte. Diese Gefässschicht ist ziemlich breit, jedoch an verschiedenen Stellen verschieden; sie ist so reich an Blut- und Lymphgefässen, dass ein Schnitt durch diese Schicht sich ausnimmt, wie eine cavernöse Membran, deren zahlreiche Höhlen die mehr oder weniger erweiterten Gefässräume darstellen, welche durch schmale Balken von Bindegewebe von einander getrennt sind. Ja, da liegt Gefäss an Gefäss, jedes mit einem Endothel versehen, so dass es auf den ersten Anblick nicht leicht wird, zu entscheiden, sind es Drüsen oder Gefässe, welche dieser Schicht einen so charakteristischen Bau verleihen. Der grösste Theil der Gefässe gehört dem Lymphsysteme an; sie sind fast alle angefüllt mit Fibrin und lymphogenen Elementen: man sieht in Fibrin eingelagert kleine und grosse Lymphocyten, die ersteren mit schön gefärbtem rundlichem Kerne, die letzteren um diesen Kern noch einen deutlichen Protoplasmasaum zeigend, so dass sie dadurch den Leucocyten und wirklichen weissen Blutkörperchen sehr nahe kommen. Diese Lymphgefässe wechseln mit feinen, stark erweiterten Capillaren ab, und wenn wir dann noch zahlreiche Blutaustritte in der Umgebung der Gefässe erblicken, so muss uns erst recht zum Bewusstsein kommen, welch ein ungeheures Ernährungsmaterial in dieser kleinen Schicht enthalten ist. Ein Blick auf Fig. 8 lehrt, dass nur wenige Gefässe leer getroffen werden und weitaus die Mehrzahl den beschriebenen Inhalt aufzuweisen hat. 
Das schmale Balkennetz, welches zwischen den Gefässen die Grundsubstanz dieser Schicht ausmacht, besteht aus Bindegewebsfasern, die durch gegenseitige Durchkreuzung ein ziemlich dichtes Gewebe darstellen. Bindegewebszellen werden nur in den tiefsten Lagen zwischen diesen Bindegewebsfasern wahrgenommen; dafür finden sich hier aber auch noch schöne, runde und ovale, kernhaltige Zellen eingelagert, die den weissen Blutkörperchen an Grösse sehr nahe kommen.

5) Unterhalb dieser bindegewebigen Gefässschicht folgt dann als untere Grenze des Chorion ein Epithel.

Auf einer schmalen Zone parallel verlaufender Bindegewebsfasern sitzt eine zweifache Lage eines cubischen Epithels auf, das aus schönen Zellen mit hübschem Kerne besteht, wie aus Fig. $7 i$ hervorgeht.

Die gefässhaltige Bindegewebsschicht stammt nach v. B a e r, Coste und v. Kölliker von der Allantois ab. Wenn diese Forscher aber weiter behaupten, dass die Allantois, sobald sie die seröse Hülle erreicht habe, nicht als Ganzes an der ganzen inneren Oberfäche der letzteren weiter wuchere, sondern blos die bindegewebige Schicht derselben mit den Blutgefässen sich der Innenfläche der serösen Hülle infolge Wucherung der Gefässschicht anlege, so muss ich dem widersprechen. Ich muss dieser Anschauung die Thatsache entgegenhalten, dass an unserem Präparat die bindegewebige Gefässschicht nach innen von einem deutlichen Epithel ausgekleidet ist, das nichts anderes sein kann, als die ursprïngliche Epithelialschicht der Allantois. Ich kann daher auch nicht v. Kölliker beipflichten, wenn er sagt, dass die Epithelialschicht der Allantois bedeutungslos verschwinde. Auch die Ansicht dieses Autors, dass die Allantois sich nicht als Blase an der Bildung des Chorion betheiligt, verliert ihre Hauptstütze durch meine unmittelbare Beobachtung, durch den Nachweis einer Epithellage zwischen Chorion und Amnion.

An dieser Thatsache kann auch der Umstand nichts ändern, dass v. Kölliker die deutlichsten Reste der Allantois in Gestalt eines, gegen die Insertion des Nabelstranges zu sich verschmälernden, Epithelialrohres gesehen hat; giebt doch dieser Forscher selbst an, dass er nur in $\mathrm{g}$ ewissen Fällen im Nabelstrange von Embryonen aus dem zweiten Monat diese blasigen Gebilde beobachtet habe. Somit lässt sich die Vermuthung nicht von der. Hand weisen, dass in diesen Fällen vielleicht Abnormitäten vorgelegen 
haben; übrigens ist diese Annahme nicht einmal unbedingt nothwendig. Es ist wohl denkbar, dass hier und da die innerste Lage des Allantoisepithels von der äusseren Lage desselben einmal durch irgend welche Wachsthumsstörungen sich lostrennt und in Gestalt eines solchen blasigen Gebildes, wie es v. Kölliker gesehen hat, noch einige Zeit besteht, ohne aber weiter zu wachsen. Daneben kann aber die äussere Lage des Epithels in ihrer ursprünglichen natürlichen Verbindung mit der Gefässschicht verbleiben und sich mit der letzteren an der Bildung des Chorion. betheiligen.

Ich muss nach dem Gesagten meine Anschauung in dieser Frage dahin ausdrücken, dass die ganze Allanto is - Gefäss blatt und Epithelialblatt - oder in den vorhin erwähnten Fällen wenigstens mit einem Theile des Epithelblattes - als Blase an der ganzen inneren Oberfläche der serösen Hülle herumwohert und die inneren Lagen des Chorion, die bindegewebige Gefässschicht und das innere Epithel oder Endothel, bildet.

Die bindegewebigen Fasern der Zotten haben jedoch mit dieser Schicht nichts zu thun, und man darf deshalb auch nicht behaupten, dass die bindegewebige Gefässschicht sich an der Zottenbildung auch nur irgendwie betheilige.

Die erwähnte Membrana limitans bildet nach meiner Ansicht die natürliche und auch entwickelungsgeschichtliche Grenze zwischen dem Theile des Chorion, welcher aus der serösen Hülle, und dem, welcher aus der Allantois hervorgeht.

Dies sind die histologischen Einzelheiten, welche ich über das Chorion laeve an meinem Präparate gewonnen habe.

Die histologische Beschreibung der Uterusmuskulatur dürfte weniger Interesse erregen, da sich über deren Bau nicht viel anderes sagen lässt, als dass sie eine gleichmässige Hyperplasie glatter Muskelfasern darstellt, welche, in allen möglichen Richtungen zu einander verlaufend, ein dichtes Filzwerk bilden.

Nur möchte ich diese kleine Arbeit nicht schliessen, ohne vorher noch einmal mit wenigen Worten auf die Decidua vera zurückzukommen.

Auf die an unserem Präparate so deutlich in die Augen springende Trennung der Decidua vera und Gebärmutterwand möchte ich nämlich an dieser Stelle noch einmal ausdrücklich hinweisen. Vielleicht darf ich an diese Thatsache eine gewisse theoretische 
Betrachtung anschliessen, welche einiges Licht verbreiten kaun über die Lösung der noch dunklen und schwierigen Frage, in welcher Schicht gewöhnlich oder in den meisten Fällen die Vera bei der regelrechten Geburt sich mit den Eihäuten losstösst, oder anders ausgedrückt, wieviel von der Decidua vera gewöhnlich bei der Geburt am regelrechten Ende der Schwangerschaft im Uterus zurückbleibt, wieviel sich mit den übrigen Eihäuten aus demselben losstösst. Ich glaube annehmen zu dürfen - um diese Annahme zur unbedingten Gewissheit zu erheben, wäre eine wiederholte ähnliche Beobachtung, wie die hier vorliegende, nothwendig - dass diese Losstossung bei der Geburt am normalen Ende der Schwangerschaft gewöhnlich in dieser, an unserem Präparate so scharf hervortretenden Trennungsschi cht sich vollzieht. Mit anderen Worten: In den meisten Fällen wird so viel von der Decidua vera mit den Eihäuten in der Nachgeburtsperiode ausgestossen werden, als ausserhalb dieser Trennungszone fällt; es dürfte also wohl die ganze Decidua vera bis auf die untere, die blinden Drüsenendigungen enthaltende, Lage der Gebärmutterschleimhaut bei dieser Gelegenheit ausgestossen werden. Es hat doch offenbar etwas Gezwungenes, anzunehmen, dass im weiteren Verlaufe der Schwangerschaft hier, in dieser Zone, eine so innige Verwachsung "zwischen der eigentlichen Decidua vera und ihrer Unterlage eintreten sollte, dass die jetzt so deutliche Trennung vollständig verwischt und die im Beginne der Schwangerschaft vorhandene Lücke durch einen bindegewebigen Kitt ausgefuillt werde, der die Festigkeit des übrigen Decidualgewebes gewinnt.

Von vornherein lässt sich viel eher Folgendes als das Wahrscheinliche in dieser Hinsicht 'darstellen. In der fünften Woche der Schwangerschaft ist die Decidua vera von dem unteren, die blinden Drüsenendigungen enthaltenden Theile der Gebärmutterschleimhaut deutlich getrennt. Dies ist keine Vermuthung, sondern eine unserem Präparate entnommene Thatsache. Mit dem allmäligen Wachsthume des Uterus und des Eies im weiteren Schwangerschaftsverlaufe wird die an unserem Präparate dicke Vera immer dünner ausgezogen und geht mit ihrer Unterlage leichtere entzündliche Verwachsungen ein, entsprechend denen, wie sie auch zwischen Vera und Reflexa vor sich gehen. So kommt es, dass die Vera später nicht mehr so locker der Gebärmutterwand einfach anliegt, wie in der fünften Woche. Auf der anderen Seite wird aber diese Verwachsung gew öhnlich nicht eine so innige werden, dass man 
כ10 Gottsehalk, Ein Uterns gravidus aus der fünften Woche u. s. w.

nicht auch später an der dünnen Haut diese vormalige Trennungszone an dem lockeren Gefüge des sie ersetzenden Gewebes stets erkennen könnte. Kommt es nun in der Nachgeburtsperiode durch die Zusammenziehungen des Uterus einerseits, den Zug der invertirten gelösten Placenta andererseits, zur Trennung der Eihäute von der Gebärmutterwand, so wird die letztere doch nothwendigerweise in der Schicht erfolgen müssen, welche obigen, hierbei wirksamen Kräften am wenigsten Widerstand zu leisten vermag, also in unserer Trennungsschicht.

Damit soll jedoch keineswegs gesagt sein, dass nicht ausnahmsweise die spätere Verwachsung zwischen Decidua und Gebärmutterwand eine so innige und also gleichsam pathologische werden kann, dass das neugebildete Gewebe in der ehemaligen Trennungszone unnachgiebiger wird, als das sonstige eigentliche deciduale Gewebe. Die Folge eines solchen Verhaltens würde dann sein, dass ein Theil, ja wenn die Vera mit der Reflexa nur eine sehr lockere Verbindung eingegangen ist, im einzelnen Falle auch die g a nze Decidua vera im Uterus zurückbleibt, und die Reflexa allein sich mit den übrigen Eihäuten losstösst.

\section{Druckfehler-Berichtignng.}

Im 2. Hefte dieses Bandes S. 337, Z. 13 v. u., muss es heissen: mit den Ovarien, statt: mit der Ovarie. 

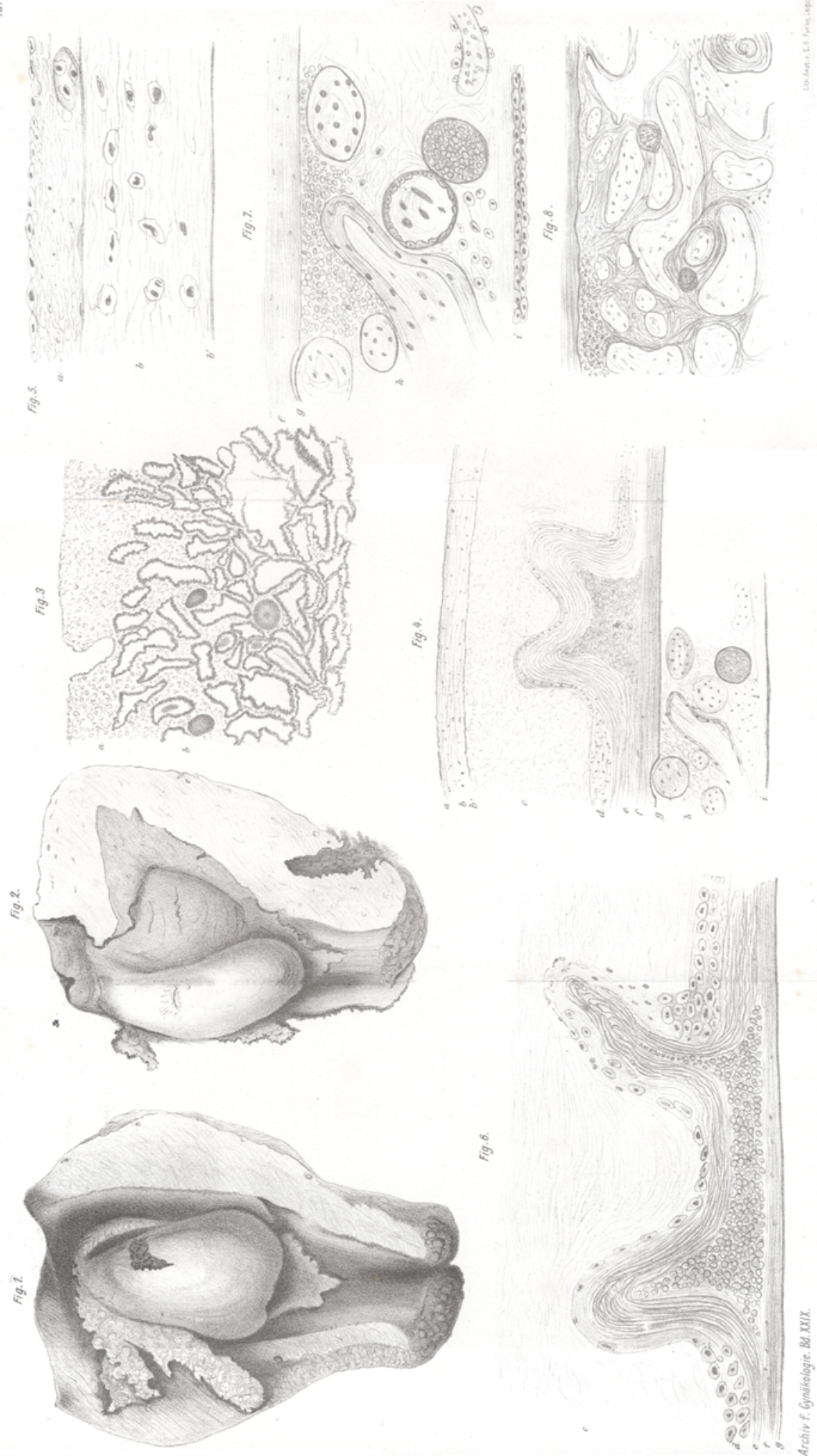